\title{
Automated selection of brain regions for real-time fMRI brain-computer interfaces
}

\author{
Citation for published version (APA):
}

Lührs, M., Sorger, B., Goebel, R., \& Esposito, F. (2017). Automated selection of brain regions for real-time fMRI brain-computer interfaces. Journal of neural engineering, 14(1), [016004]. https://doi.org/10.1088/1741-2560/14/1/016004

Document status and date:

Published: 01/02/2017

DOI:

10.1088/1741-2560/14/1/016004

Document Version:

Publisher's PDF, also known as Version of record

Document license:

Taverne

\section{Please check the document version of this publication:}

- A submitted manuscript is the version of the article upon submission and before peer-review. There can be important differences between the submitted version and the official published version of record.

People interested in the research are advised to contact the author for the final version of the publication, or visit the DOI to the publisher's website.

- The final author version and the galley proof are versions of the publication after peer review.

- The final published version features the final layout of the paper including the volume, issue and page numbers.

Link to publication

\footnotetext{
General rights rights.

- You may freely distribute the URL identifying the publication in the public portal. please follow below link for the End User Agreement:

www.umlib.nl/taverne-license

Take down policy

If you believe that this document breaches copyright please contact us at:

repository@maastrichtuniversity.nl

providing details and we will investigate your claim.
}

Copyright and moral rights for the publications made accessible in the public portal are retained by the authors and/or other copyright owners and it is a condition of accessing publications that users recognise and abide by the legal requirements associated with these

- Users may download and print one copy of any publication from the public portal for the purpose of private study or research.

- You may not further distribute the material or use it for any profit-making activity or commercial gain

If the publication is distributed under the terms of Article $25 \mathrm{fa}$ of the Dutch Copyright Act, indicated by the "Taverne" license above, 


\title{
Automated selection of brain regions for real-time fMRI brain-computer interfaces
}

\author{
Michael Lührs ${ }^{1,2}$, Bettina Sorger ${ }^{1,2}$, Rainer Goebel ${ }^{1,2,3}$ \\ and Fabrizio Esposito ${ }^{1,4}$ \\ ${ }^{1}$ Department of Cognitive Neuroscience, Maastricht University, Maastricht, The Netherlands \\ 2 Maastricht Brain Imaging Center, 6229 ER Maastricht, The Netherlands \\ 3 Netherlands Institute for Neuroscience (NIN), 1105 BA Amsterdam, The Netherlands \\ ${ }^{4}$ Department of Medicine, Surgery and Dentistry, Scuola Medica Salernitana, University of Salerno, \\ Baronissi (SA), Italy \\ E-mail: faesposito@unisa.it
}

Received 10 May 2016, revised 6 November 2016

Accepted for publication 8 November 2016

Published 30 November 2016

\begin{abstract}
Objective. Brain-computer interfaces (BCIs) implemented with real-time functional magnetic resonance imaging (rt-fMRI) use fMRI time-courses from predefined regions of interest (ROIs). To reach best performances, localizer experiments and on-site expert supervision are required for ROI definition. To automate this step, we developed two unsupervised computational techniques based on the general linear model (GLM) and independent component analysis (ICA) of rt-fMRI data, and compared their performances on a communication BCI. Approach. $3 \mathrm{~T}$ fMRI data of six volunteers were re-analyzed in simulated real-time. During a localizer run, participants performed three mental tasks following visual cues. During two communication runs, a letter-spelling display guided the subjects to freely encode letters by performing one of the mental tasks with a specific timing. GLM- and ICA-based procedures were used to decode each letter, respectively using compact ROIs and whole-brain distributed spatio-temporal patterns of fMRI activity, automatically defined from subjectspecific or group-level maps. Main results. Letter-decoding performances were comparable to supervised methods. In combination with a similarity-based criterion, GLM- and ICA-based approaches successfully decoded more than $80 \%$ (average) of the letters. Subject-specific maps yielded optimal performances. Significance. Automated solutions for ROI selection may help accelerating the translation of rt-fMRI BCIs from research to clinical applications.
\end{abstract}

Keywords: ICA, GLM, BCI, rt-fMRI, communication BCI, neurofeedback, ROI selection

(Some figures may appear in colour only in the online journal)

\section{Introduction}

Real-time functional magnetic resonance imaging (rt-fMRI) allows immediate access to brain activity information by analyzing blood oxygen level dependent (BOLD) activation signals as fast as they are acquired [1]. For this reason, rtfMRI can be used as a brain-computer interface (BCI) with high spatial resolution and whole-brain coverage, thereby overcoming some of the limitations of electroencephalography (EEG) based BCIs [1]. Rt-fMRI BCIs can also be used for neurofeedback [1-4]. In these applications, the rt-fMRI $\mathrm{BCI}$ is expressly designed to implement the real-time feedback of BOLD signals to the experimental subject or the patient. Thereby, researchers and clinicians can use rt-fMRI BCIs to train individual subjects to self-regulate their brain activity in various different brain areas, and possibly observe 
Table 1. Participants' characteristics. The table provides information concerning the participants' age, sex, handedness (as evaluated by the Edinburgh inventory [18]), and prior fMRI experience. Moreover, it is indicated how many volumes were acquired in each functional run. Adapted from [9].

\begin{tabular}{|c|c|c|c|c|c|c|c|}
\hline \multirow[b]{2}{*}{ Participant } & \multirow[b]{2}{*}{ Age (years) } & \multirow[b]{2}{*}{ Sex } & \multirow[b]{2}{*}{ Handedness } & \multirow{2}{*}{$\begin{array}{l}\text { Number of prior } \\
\text { fMRI sessions }\end{array}$} & \multicolumn{2}{|c|}{$\begin{array}{l}\text { Functional volumes } \\
\text { scanned }\end{array}$} & \multirow{2}{*}{$\begin{array}{l}\text { Performed } \\
\text { mental task }\end{array}$} \\
\hline & & & & & Initial run & Follow-up run & \\
\hline $\mathrm{S} 1$ & 37 & Female & Right & 33 & $472 / 556$ & $682 / 338$ & MI, MC, IS \\
\hline $\mathrm{S} 2$ & 33 & Female & Right & 21 & 472 & 388 & MI, MC, MS \\
\hline S3 & 29 & Female & Right & 13 & 304 & 430 & MI, MC, IS \\
\hline $\mathrm{S} 4$ & 22 & Male & Left & 3 & 514 & 430 & MI, MC, IS \\
\hline S5 & 25 & Male & Right & 1 & 304 & 304 & MI, MC, IS \\
\hline S6 & 30 & Female & Left & 21 & 430 & 472 & MI, MC, IS \\
\hline
\end{tabular}

Remarks: $\mathrm{MI}=$ motor imagery, $\mathrm{MC}=$ mental calculation, $\mathrm{IS}=$ inner speech, $\mathrm{MS}=$ mental singing.

specific behavioral or therapeutic effects across repeated sessions of the experiment [1-4].

Although fMRI is more costly than EEG, rt-fMRI BCIs may offer unique opportunities, especially in the field of communication and control BCIs [5]. For instance, in recent studies, it has been shown that participants were able to control brain activation within different brain regions distinctively, e.g. to answer multiple-choice questions or to navigate through a virtual maze [6-8]. A more recent study [9] introduced a motor-independent communication technique that allowed decoding up to 27 distinct information units. The communication BCI introduced by Sorger and colleagues [9] enables bi-directional communication using BOLD signals in predefined brain regions of interest (ROIs). In fact, using this letter-spelling rt-fMRI BCI, participants were able to communicate freely chosen answers to open questions.

From a technical standpoint, both rt-fMRI communication/ control and neurofeedback BCIs rely on a general rt-fMRI analysis pipeline, thereby similar issues pertain to, and common solutions are shared between, the two applications. Among these, the selection of the ROIs, as either BCI signal source(s) or neurofeedback target location(s), is considered critical for both BCI decoding performances and for neurofeedback treatment success $[2,10]$. In previous rt-fMRI works, depending on the specific application, source/target ROIs have been typically obtained using (i) available anatomical atlases (see, e.g. [11]), (ii) thresholded group-level functional maps from separate fMRI experiments (see, e.g. [12]) or (iii) subject-specific functional maps from within-session localizer experiments (see, e.g. [13]).

In order to reach best possible decoding and neurofeedback performances, the selection of ROIs in fMRI-based BCIs is usually optimized within the specific application. This can be achieved with carefully designed functional localizer experiments prior to the actual BCI experiment. However, in this case, multi-domain cognitive expertise (especially about functional network topographies) as well as on-line supervision capabilities to manually set appropriate statistical models and thresholds are crucial to optimally select the relevant task-related brain regions from localizer data sets. These aspects may impede the use of fMRI-based BCIs in clinical setups due to the need of human experts. In addition, because fMRI responses and patterns may change significantly in patients, due to, e.g. unknown levels of cognitive capacities, attention, arousal and communication intents (see, e.g. [14]), the same spatial and temporal models used for a given setup in healthy volunteers, might not work with identical performances in clinical settings.

In an attempt to automatize the procedure of selecting fMRIbased BCI signal sources, we developed two novel unsupervised computational approaches, one based on the standard general linear model (GLM) [15] and one based on real-time independent component analysis (ICA) [16], and (re-)analyze data presented in [9] and an additional data set, in a simulated real-time framework. Although the proposed methods were applied in off-line data analyses of existing data, which could in part limit the general validity of the presented results, we present a novel and fully automatic processing pipeline that can be used as is in an actual real-time setup.

We hypothesized that using the proposed selection strategies and algorithms, comparable performances between the proposed unsupervised solutions and the previous supervised solutions could be achieved. Because our initial simulations clearly indicated that the implemented pipeline is fully compatible with real-time applications, the results obtained with the presented approaches can be useful for the implementation of a fully automated rt-fMRI BCI. More in general, these approaches could be applied in other communication/control and neurofeedback BCIs, e.g. to enhance effective connectivity [11], to develop new experimental paradigms in cognitive neuroscience [12] or for closed-loop training [17].

\section{Methods}

\subsection{Description of the $f M R I$ data sets}

(F)MRI data of six participants (mean age $29.3 \pm 5.39$ years, four female and two male) were re-analyzed. Details about the population can be found in table 1, including participants' age, sex, handedness (as evaluated by the Edinburgh Inventory [18]), and prior fMRI experience. Moreover, it is indicated how many volumes were acquired in each functional run [9]. For subject S1 data of two sessions were available, of which the second data set was not included in the previous study [9].

\subsection{Description of the $\mathrm{BCl}$ and experimental design}

The background of the letter encoding technique and all details about the experimental design can also be found in [9]. For the 


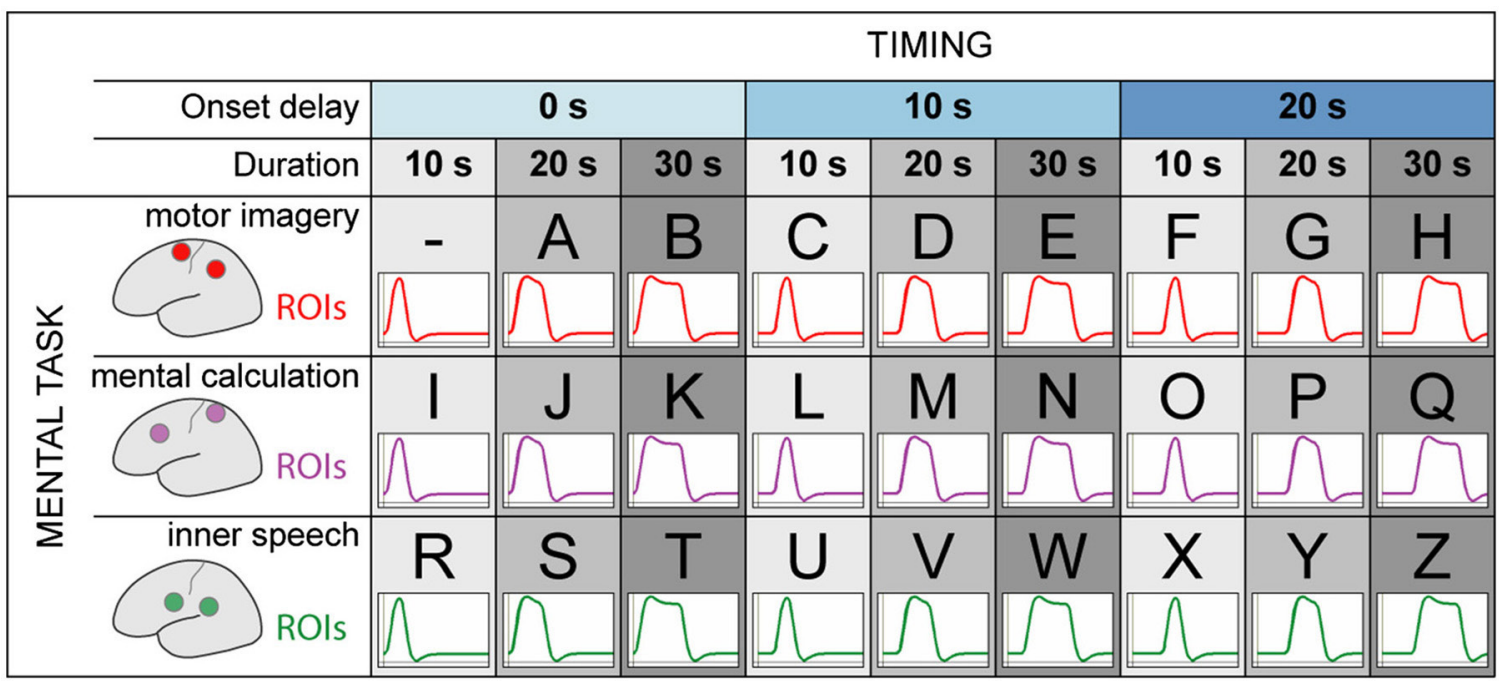

Figure 1. Letter coding scheme. Combining three mental tasks, three task onset delays, and three task durations allows for encoding $3 \times 3 \times 3=27$ distinct information units. When assigning these 27 combinations to 26 letters and the blank space ('-'), every character can be represented by a certain cognitive event leading to a unique dynamic brain response pattern. In order to encode, e.g. the letter 'E', a motor action has to be imagined, starting $10 \mathrm{~s}$ after the general trial onset and lasting for $30 \mathrm{~s}$. Remarks/abbreviations are as follows: RTCs, reference time courses; ROIs, regions of interest; see table S1 for mental tasks and ROIs used in the current study; see [9] Movie S1 for brain activation patterns evoked by performing different mental tasks; curves shown are modeled RTCs derived from standard hemodynamic response functions (note, however, that individual RTCs were used for letter decoding (see [9] figure S3 for comparison of individual versus standard RTCs)). Figure reused from [9] with permission by Elsevier. Copyright 2012.

sake of comprehension of the present paper, the description of the BCI has been repeated here.

In order to represent each letter of the English alphabet and the blank space by a distinct neural activation pattern, a letter encoding technique was implemented that requires almost zero pretraining. This technique exploits spatiotemporal characteristics of single-trial BOLD signals that are associated with the participant's performance of different mental tasks and certain temporal aspects of task execution. That a single cognitive event can be assessed online has been demonstrated by Posse and colleagues [19]. To intentionally generate 27 distinguishable hemodynamic activation patterns, participants voluntarily influenced three BOLD signal aspects: (i) location of the signal source by performing three different mental tasks, (ii) signal onset delay by delaying the start of the mental task for $0 \mathrm{~s}, 10 \mathrm{~s}$, or $20 \mathrm{~s}$, and (iii) signal duration by varying the mental task duration between $10 \mathrm{~s}, 20 \mathrm{~s}$, and $30 \mathrm{~s}$. Full combination of these parameters results in the generation of 27 unique hemodynamic brain responses, which can be assigned to 27 characters (figure 1). The letter-by-letter translation of any freely chosen answer into single-trial BOLD signals required only little effort from participants as the encoding process was facilitated by using a convenient visual letter encoding display designed according to the coding scheme (figure 1). For encoding a certain letter, participants merely had to attend to the desired letter and to perform the designated mental task as long as the letter was highlighted in the display (figure 2).

After each letter encoding trial, the participants had to passively view the display and rest until the next trial. During this trial, no feedback was given to the participants. According to previous (EEG-based) BCI studies (see, e.g. [20, 21]), both motivation and frustration can be increased by feedback and error monitoring could lead to changes in BCI performances. In this case, it was decided to not give letter by letter feedback to prevent frustration, also considering that not every letter needs to be correct in order to later be able to successfully interpret the whole word.

In a localizer experiment preceding the experimental runs, the participants were instructed to perform one of the three different tasks as soon and for as long as the word of the task (e.g. motor imagery) was shown on the display. To later derive individual response functions for each participant, the three different tasks where shown for three different time intervals $(10,20$ and $30 \mathrm{~s})$.

\subsection{MRI data acquisition}

FMRI data were acquired with a 3 T MRI head scanner (Magnetom Allegra, Siemens Healthcare, Erlangen, Germany) using a quadrature birdcage head coil. In all sessions, a single-shot repeated echo-planar imaging (EPI) sequence was used to produce BOLD-fMRI image time series. Except for the number of acquisitions (localizer experiment: 372 volumes; for further details, see table 1), identical scanning parameters were used for both experimental runs (repetition time $(\mathrm{TR})=2000 \mathrm{~ms}$, echo time $(\mathrm{TE})=30 \mathrm{~ms}$, flip angle $(\mathrm{FA})=90^{\circ}$, field of view $(\mathrm{FOV})=224 \times 224 \mathrm{~mm}^{2}$, matrix size $=64 \times 64$, number of slices $=34$, slice thickness $=3.5 \mathrm{~mm}$, no gap, slice order $=$ ascending/interleaved).

For each participant, a three-dimensional (3D) T1-weighted data set encompassing the whole brain was acquired following the localizer experiment (scan parameters: $\mathrm{TR}=2250 \mathrm{~ms}$, $\mathrm{TE}=2.6 \mathrm{~ms}, \quad \mathrm{FA}=9^{\circ}, \quad \mathrm{FOV}=256 \times 256 \mathrm{~mm}^{2}, \quad$ matrix size $=256 \times 256$, number of slices $=192$, slice thickness $=1 \mathrm{~mm}$, total scan time $=8 \mathrm{~min}$ and $26 \mathrm{~s}$ ). Parameters of this anatomical MRI sequence were based on the Alzheimer's disease Neuroimaging Initiative protocols. Anatomical and functional MRI off-line data processing was performed using 


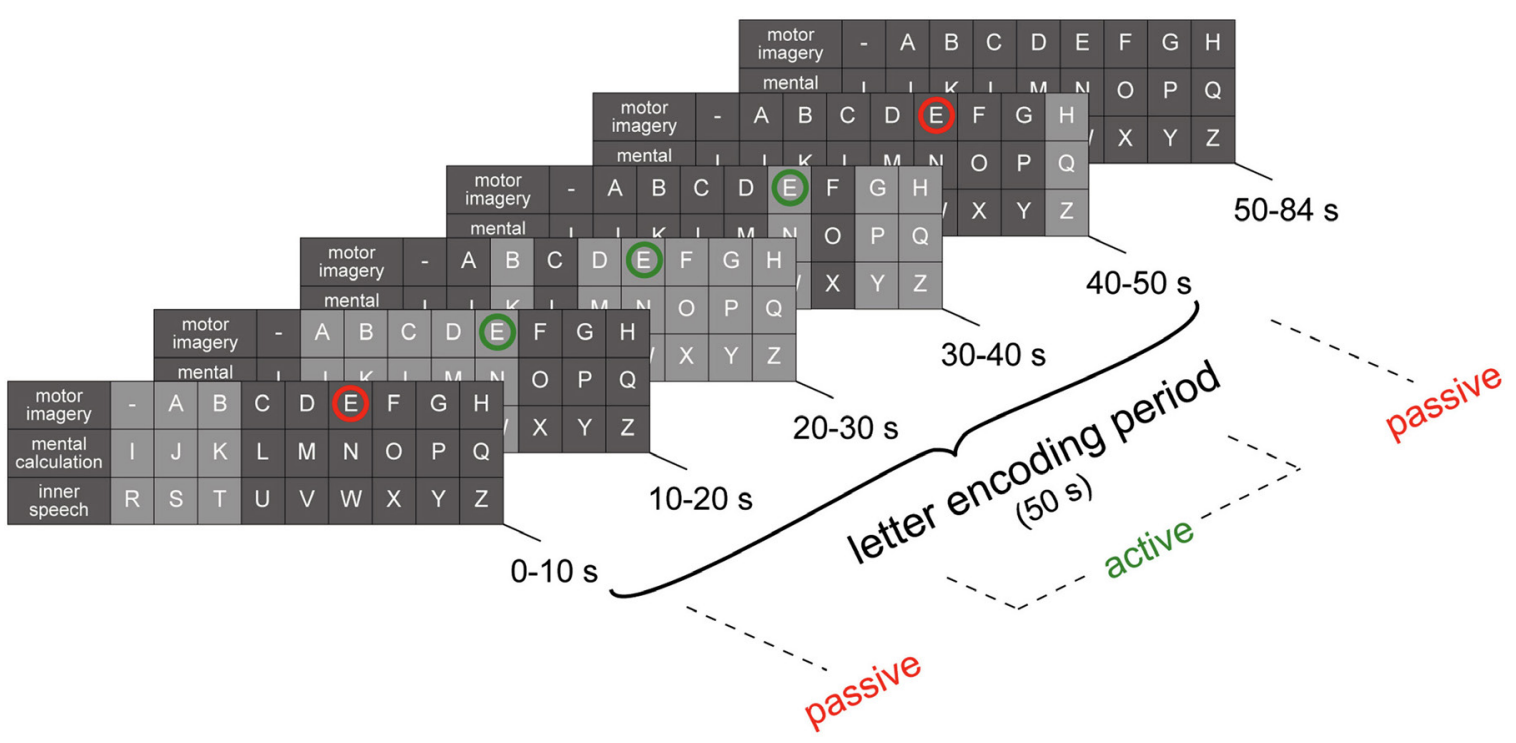

Figure 2. In order to encode a specific letter, participants did not have to memorize the encoding parameters for a certain letter (i.e. mental task, onset delay, task duration). They only had to attend to the selected letter and to perform the particular mental task assigned to the respective row (indicated in the first column of each window) as long as the accordant letter cell was highlighted (for $10 \mathrm{~s}, 20 \mathrm{~s}$, or $30 \mathrm{~s}$ ). In order to encode, e.g. the letter ' $E$ ', no mental task has to be performed in the first 10 s following the onset of the letter encoding trial (passive). When the 'E' cell gets highlighted, the participant starts performing 'motor imagery' (active). As soon as the letter cell is no longer highlighted (after $30 \mathrm{~s}$ in this example), the participant stops and stays focusing on ' $\mathrm{E}$ ' for another $10 \mathrm{~s}$ (passive) until the whole letter encoding period is finished. During the subsequent resting period, in which none of the letter cells is highlighted, the participant is asked to select (and switch to) the next intended letter remaining in a sort of 'stand-by' mode, selecting the next mental task to be performed, and awaiting the next active letter encoding phase. Note that the conceptualization of our letter encoding display and the visual display invented in the context of the development of an EEG-based spelling device via event-related potentials (P300) [22] share common characteristics. Remarks are as follows: circles were not visible for participants; they are shown here in order to emphasize active (green) and passive (red) answer encoding phases when encoding the letter 'E'. Figure reused from [9] with permission by Elsevier. Copyright 2012.

Brain Voyager QX (v2.8.4; Brain Innovation B.V., Maastricht, The Netherlands). Further details about the MRI data acquisition protocol can be found in [9].

\subsection{MRI data analysis}

Image data preprocessing (motion correction, spatial smoothing with gaussian kernel FWHM $6 \mathrm{~mm}$ ) and simulated real-time analyses were performed using Turbo-BrainVoyager (v3.2; Brain Innovation B.V., Maastricht, The Netherlands). No trend removal (high-pass filtering) was applied on the motion-corrected EPI time series to simulate the real-time analysis framework but added as a confound predictor to the GLM analysis.

The automated decoding procedures (see details below) for the ICA-based algorithm (section 2.6) was implemented in Matlab (The MathWorks, Inc., Natick, Massachusetts, USA) using the output of Turbo-Brain Voyager and the rt-ICA plugin of Turbo-BrainVoyager, that respectively consisted of the ROI time-courses and the ICA component maps and representative time-courses.

Image data spatial normalization and template ROI definitions were performed using BrainVoyager QX (v2.8.4 Brain Innovation B.V., Maastricht, The Netherlands).

\subsection{GLM-based letter decoding}

The fMRI data of the functional localizer run were used to determine the ROIs for subsequent communication runs. A standard convolution-based GLM analysis was performed on the localizer datasets using three box-car functions for the three tasks. Using two different linear contrasts calculated from the fitted GLM beta weights, two ROIs were automatically selected for each task: The first region was based on the main effects of the task (contrast: task versus rest). The second region was more specific for the task as it was based on the balanced differential effects, e.g. for task 1 the contrast was selected as task 1 $(+2)$, task $2(-1)$, task $3(-1)$. The choice of the two contrasts was motivated by the need of jointly accounting for both the generalized activation evoked by the task (main effects independent of the other tasks) and the differential effects of each specific task relative to the other tasks.

The unsupervised selection procedure for each ROI from the GLM t-maps consists of multiple steps. Starting from the GLM map obtained by a given contrast (t statistics), the algorithm adaptively searches for a t statistic threshold such that at least twenty voxels are selected forming one or more compact clusters of at least five voxels. A compact cluster is obtained from a set of adjacent voxels, where two voxels are considered adjacent if they are directly connected (i.e. they touch each other) at one of their faces, edges or vertices. Because the simple iterative search among many thresholds would imply a new search of all the clusters for each new threshold, to avoid repeating the search for all thresholds, we used an interpolation search method [23]. This algorithm searches for a given value in a sorted and indexed list with on average $O(\log (\log (n)))$ comparisons and can be implemented with the following code (adapted from [23]): 


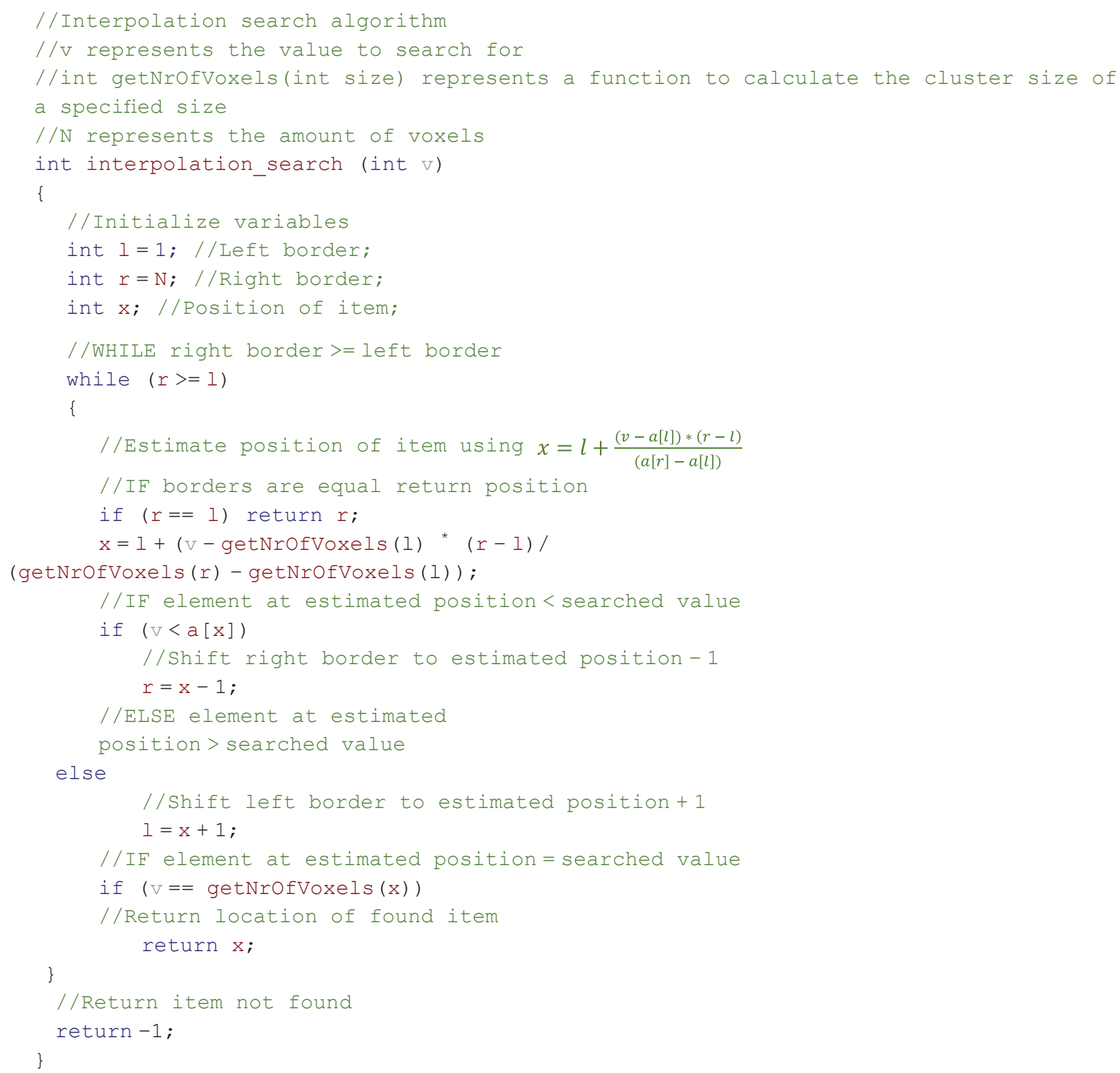

In this way, two sets of voxels (due to the above two contrasts) were independently determined for each task, albeit it was not prohibited that (i) more than one compact region was included in the final (multi-)ROI as a cluster assembly or network and (ii) all cluster assemblies ultimately consisted of the same voxels (in total or in part).

Apart from ROI selection, the automated letter-decoding procedure was the same as used in the previous study, ranking all 27 characters according to their overall fit with a combined letter-decoding criterion incorporating both shape and amplitude-pattern information. The shape criterion is obtained by the correlation of the ROI time courses with a multi-filter bank of hemodynamic response functions; the amplitude pattern criterion is obtained by the regression of the ROI timecourses on the multiple hemodynamic response functions and the extraction of the estimated single-trial beta weights within all six ROIs [9]. For calculating both shape and amplitudepattern scores according to this criterion, the hemodynamic response functions could either be ideal response functions or individually derived empirical responses generated from the mean ROI time courses in the localizer run, thereby both response function types were explored in our tests. The ideal response functions were derived by linearly convolving the nine box-car functions ( 3 different onset delays $\times 3$ different stimulus durations) with the double-gamma hemodynamic response function [24].

To further investigate the potentials of the approach, especially in the direction of localizer-free experimental settings, the selected ROIs of each participant were transformed into Talairach space and combined into group-level (template) ROIs. To test the performances of the GLM-based decoding using template ROIs, a leave-one-out approach was used in which the spatially normalized (Talairach-transformed) ROIs were merged from all participants except the participant that was investigated during decoding and then back-transformed to the native space of this participant and given as an input for the decoding algorithm.

All Talairach transformations were estimated on the individual anatomical images using 12-parameter affine transformations, and applied to the functional images. 


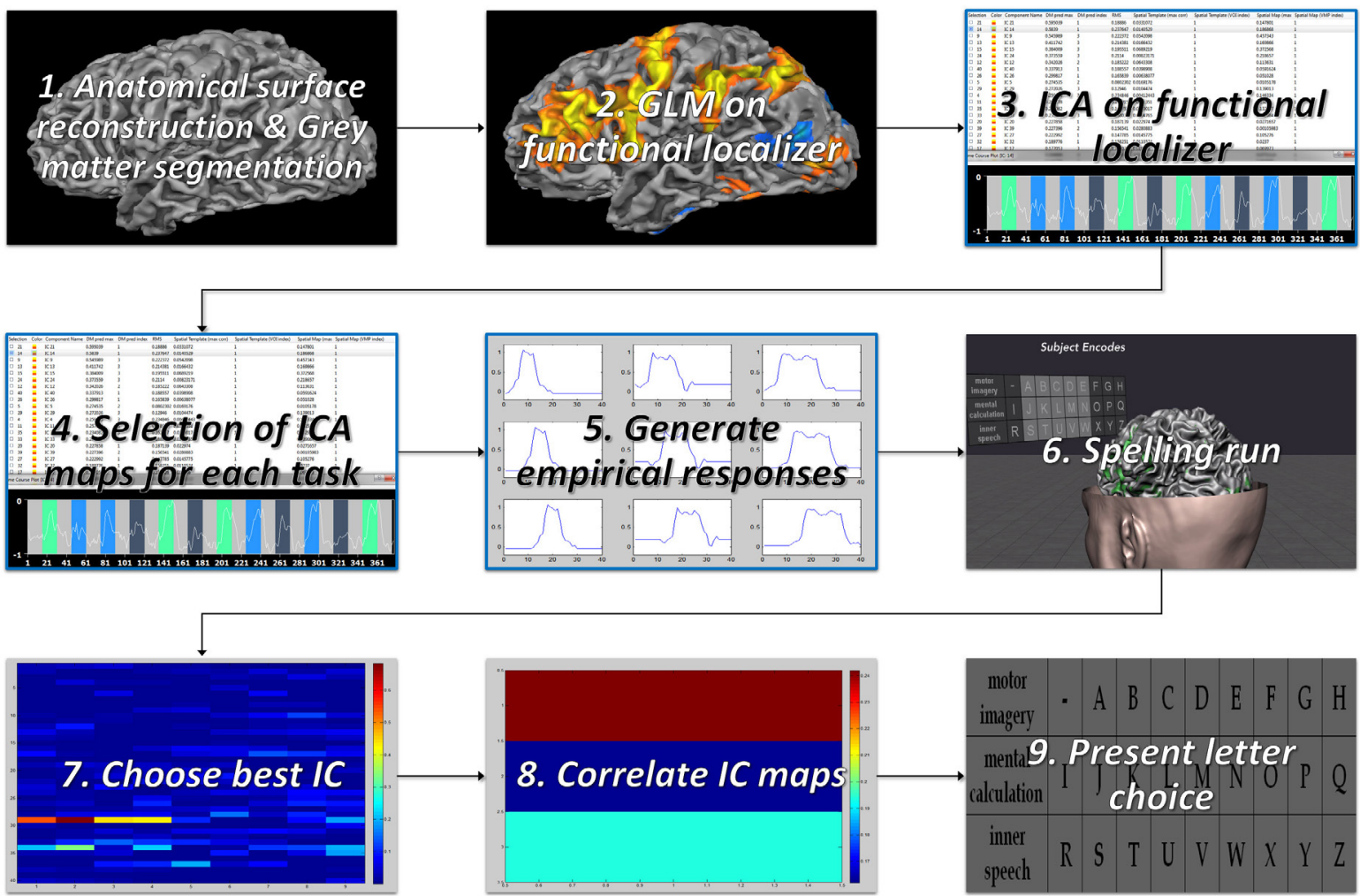

Figure 3. ICA letter decoding algorithm scheme. The ICA based decoding algorithm consists of nine iterative steps that are performed after each other. Steps three to five, marked with a blue border, are only necessary for implementing the empirical approach with real-time ICA. In a first step the anatomical surface reconstruction and a grey matter segmentation is performed to run ICA only on grey matter voxels. Afterwards reference GLM maps from the functional localizer run of each session are prepared. In step three the whole functional localizer is analyzed using ICA. For each task separate ICA maps are extracted based on the highest correlation with the GLM maps prepared in the previous step. These maps were later used in step eight. The following step generates empirical responses from the ICA time courses combined of each selected task in the prior step. After the preparation steps the real spelling run can be performed. Using the acquired single trial data for each letter the specific independent component is selected. In step eight the IC map of the selected component is correlated with the maps prepared in the localizer. The last step presents the letter choice to the experimenter.

In more detail, each anatomical data set was first transformed to the bi-commissural (ACPC) space using a single affine transformation and then warped to Talairach standard space using a piece-wise grid-scaling affine transformation. The individual ROIs, initially obtained as voxel masks in the native space of functional data sets, were transformed to Talairach space using the same parameters obtained from the anatomical data sets after careful functional-to-anatomical image alignment. In order to avoid quality loss due to successive data sampling, functional-to-anatomical image registration and normalization were performed in one single step by combining the estimated functional-anatomical affine transformation, the rigid-body ACPC affine transformation and the piecewise affine Talairach grid scaling. The merging of all individual ROIs (both within and between subject) into a group-level (template) ROI was performed in the normalized space and consisted in the logical OR of all transformed masks. As the combined spatial transformations were also applied backward in one step and concatenated to form a single $4 \times 4$ affine transformation matrix, the obtained coordinates of the group-level (template) ROI voxels in the normalized space were used to calculate the coordinates of the group-level (template) ROI voxels in the original 3D space of each subject, allowing to precisely sample the data points in the functional data set via sinc interpolation.

\subsection{ICA-based letter decoding}

The novel ICA letter-decoding method consists of nine consecutive calculation steps to determine the encoded letter (figure 3). First, surface reconstruction is applied to the anatomical data set which was acquired for each participant at the beginning of each session. This step requires the correction of inhomogeneities of the MRI signal intensity across space, the white/gray matter border segmentation (performed with a region-growing method using intensity histograms), a number of morphological operations to smooth the borders of the segmented data and to separate the left from the right hemisphere, and the creation of a triangular mesh representing the white/ gray matter border surface. This mesh is then projected into the functional data, thereby those voxels that lie within a standard range with respect of the white/gray matter border (usually from $1 \mathrm{~mm}$ inward to $3 \mathrm{~mm}$ outward) are tagged as 'gray matter' voxels and define the gray matter masks to be used in the simulated real-time ICA analyses (for more details see $[25,26])$ (step one). GLM activation maps for each task were 
calculated from the functional localizer data by contrasting each task separately from each other compared to rest (step two). The resulting activation patterns for the three tasks were taken as whole-brain maps (without thresholds) and stored for the later selection step to determine (via spatial correlation with ICA component maps) which task was performed by the participant. In step three, the whole functional localizer data set is decomposed into spatial ICA components using off-line ICA with the fastICA algorithm [27]. Prior to the standard ICA decomposition, the initial dimension of the functional dataset is reduced to 40 using principal component analysis (PCA). The PCA stage is included in standard ICA methodology and the chosen final dimensionality corresponds to more than $20 \%$ of the time points, and accounts for more than $99.9 \%$ of the total variance-covariance, of the localizer data sets [25]. For each task multiple ICA components are selected based on the highest spatial correlation between the ICA components and the task-specific GLM maps from the previous step (step four). The selected ICA components' maps and representative timecourses are used as alternative maps for selection (see below) and to calculate empirical responses via trial-based averaging (step five). All analysis steps from one to five involving the anatomical and the localizer functional data sets were performed using BrainVoyager QX (v2.8.4 Brain Innovation B.V., Maastricht), and are not intended to be part of the final simulated real-time analysis. In fact, these steps could be in principle performed in a different session, separated from the communication runs. In our simulations, step one never took more than $10 \mathrm{~min}$ on a computer equipped with a quad core processor @ $3 \mathrm{GHz}$ and $8 \mathrm{~GB}$ of RAM, and therefore can be completed by running a script during the calibration run (that lasts $12.4 \mathrm{~min}$ ). Moreover, because calibration data can be preprocessed on-line, and the analysis in steps two and three are performed in the native space, step two (GLM on calibration data) and steps three, four and five (off-line ICA + component selection based on GLM maps + empirical response function generation) could be all completed in about $3 \mathrm{~min}$ on the same computer. This suggests that all preliminary calculations can be completed before the on-line run without excessive delay between the calibration and the on-line runs.

Steps from six to nine are part of the final simulated realtime analysis of the communication runs.

Step six pertains to the letter encoding process. For the real-time ICA, the time window was set to 40 time points (covering the duration of each letter encoding trial including the rest period between two successive trials) and the calculations were started at the end of each block. Because spatial smoothing improves the detection power of the ICA procedure [16], a spatial smoothing with gaussian kernel of $6 \mathrm{~mm}$ FWHM is applied on-line to the functional data. No further pre-processing is done prior to the real-time ICA computation and a $T \times V$ data matrix is filled by taking the $V$ voxel values corresponding to the $T$ time points in the current time window as soon as these are available for real-time processing. This matrix is fed into the real-time ICA algorithm.

In real-time ICA, the number of ICA components can be set to the number of time points of the data [16]. Although it would be possible to reduce the temporal dimensions of the data prior to ICA (as is normally done in off-line ICA), this reduction is not needed for real-time ICA due to the small number of time points. Conversely, it is more important (compared to off-line ICA) to use the entire variance/covariance of the data in the time window and extract as many ICA components as possible, to avoid missing some informative components in the data. In our simulations, after each completed letter encoding trial, 40 independent components were all available for subsequent processing (steps seven and eight) within about $4 \mathrm{~s}$. After extraction, each ICA component timecourse is used in a parallelized multiple linear regression analysis with nine standard hemodynamic reference functions. Thereby, letting $\boldsymbol{y}(t)$ denote the column vector with a single component time-course, $\boldsymbol{X}=\left[\boldsymbol{x}_{1}(t), \boldsymbol{x}_{2}(t), \ldots, \boldsymbol{x}_{9}(t)\right]$ the matrix with the nine reference functions as column vectors and $\boldsymbol{b}$ a column vector with nine unknown weights, the linear model $\boldsymbol{y}=\boldsymbol{X} \cdot \boldsymbol{b}$ is least-square fitted, and the coefficient of determination $\left(r^{2}\right)$ is obtained, for each component.

The standard hemodynamic reference functions could be either ideal (i.e. derived from the two-gamma hemodynamic response model [24]) or individually determined from the empirical responses in step five. Both response models were explored in this approach. After selecting the ICA component with the highest coefficient of determination $\left(r^{2}\right)$ (step seven), the spatial correlation coefficients between the corresponding ICA component map and the three GLM-based statistical maps from step three (or the ICA maps from step four) were calculated to identify which task was most probably performed during the letter-encoding trial (step eight). Finally, the most probable participant's letter choice was determined based on the results of the previous steps (step nine).

Given the stochastic nature of the ICA algorithm, to investigate the reliability of the ICA results [28] (i.e. the stability of the point of convergence), this analysis procedure was applied five times for each encoded letter with random initial conditions. In fact, the fastICA algorithm estimates the parameters of each component (component 'weights') iteratively, returning a new component when the next iteration does not change the sum of squares of the weights more than a given tolerance (set by default to $10^{-6}$ ). Because these parameters are best initialized with random numbers, we wanted to check that repeating the simulations on the same data would not result in different decoded letters. In practice, because the important aspect in this context is not the exact point of convergence but the actual decoded letter, this step is not needed in the real-time application and therefore does not affect the on-line feasibility of the proposed approach.

Similarly to what has been done for the GLM approach, to further investigate the potential of the ICA approach with respect to a possible localizer-free experimental setting, the described algorithm was modified by removing steps one to three, and using a group-level GLM to generate a mean GLM map of all six participants (excluding the participant that was analyzed). To calculated this map, a fixed-effects GLM group data model was used to retain the highest statistical power in the studied group of participants. These template GLM maps replaced in this way the individual participant GLM maps in step eight. 


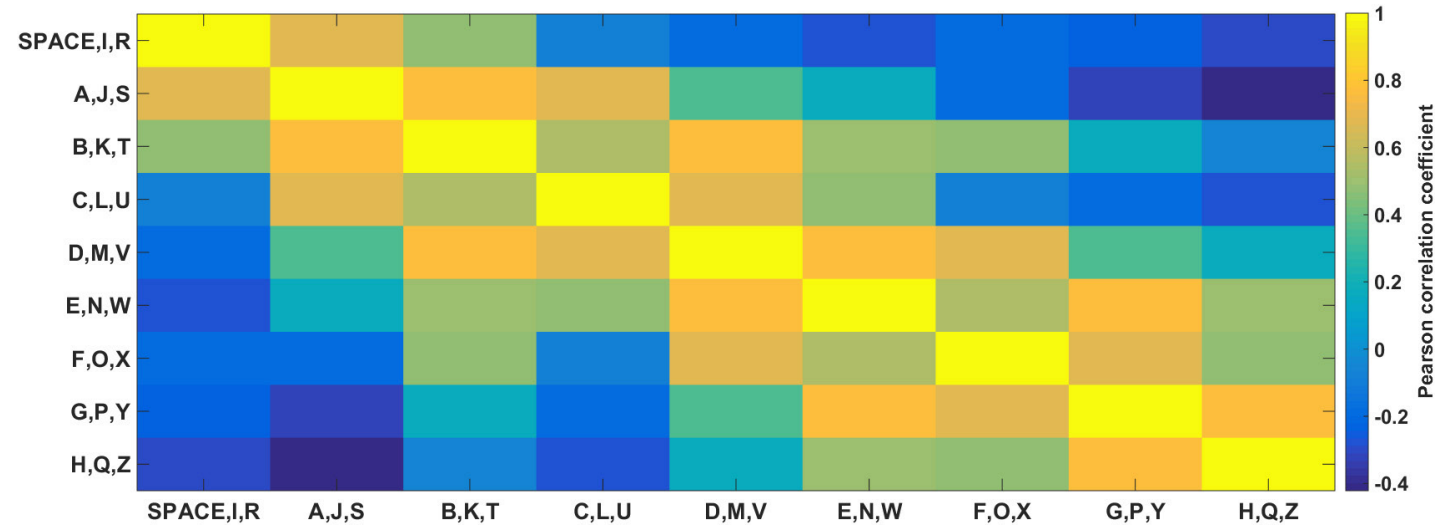

Figure 4. Correlation coefficients of the ideal response functions of each of the nine different on/offsets and lengths. For the similarity criterion the two letters having the highest correlation to the selected letter were accepted as correct choice. E.g. for letter choice ' $D$ ' the letters ' $E$ ' and 'B' would fulfil the criterion. The correlation coefficients show that letters that are very close on the letter display are not automatically the letters that have the highest correlation.

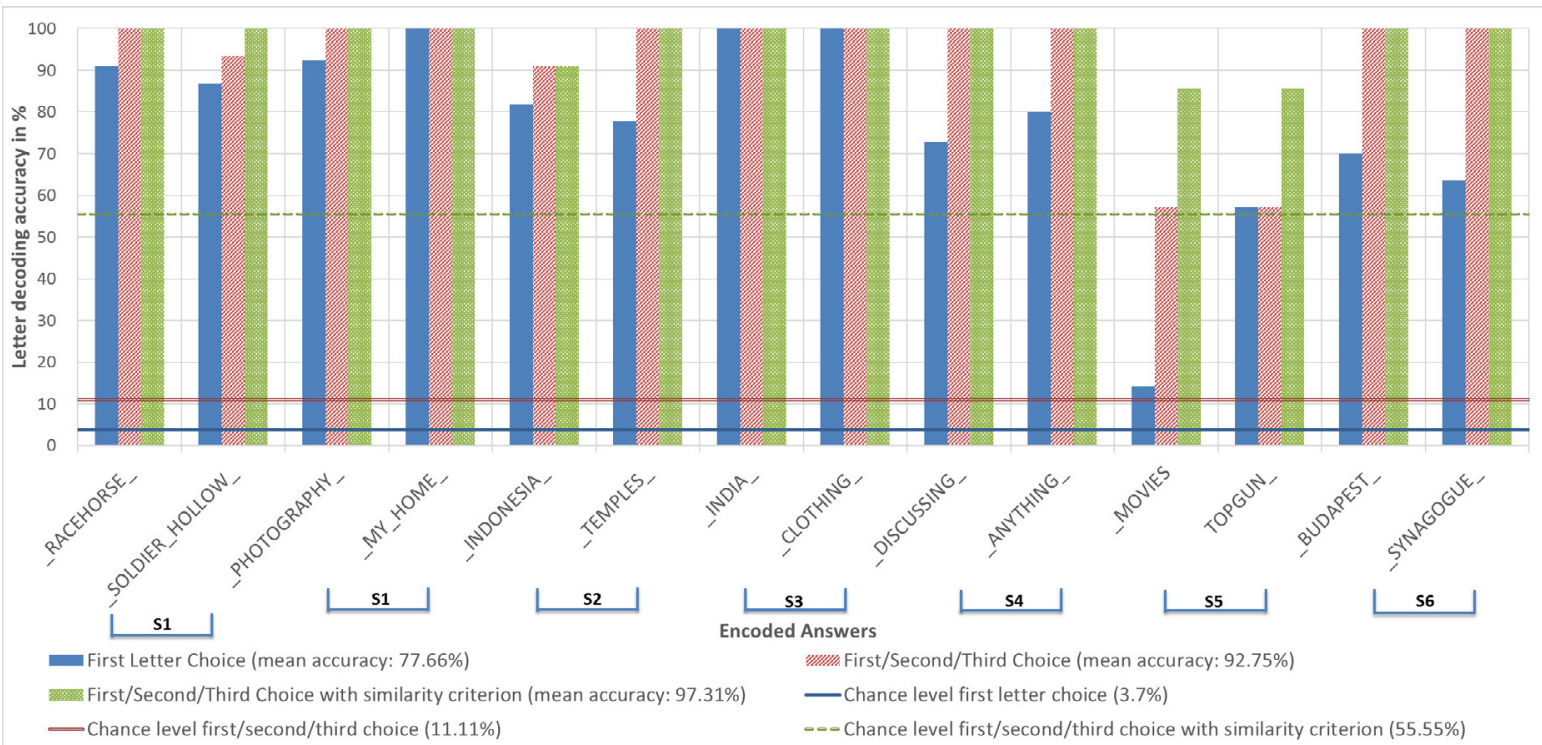

Figure 5. Letter decoding results of GLM-based approach. For each participant, the average decoding accuracy across all letters of the two individually decoded words is shown in the figure. The empirically derived response functions were used in the shown results.

\subsection{Decoding performance evaluation}

In order to assess and compare the performances of the proposed solution, a similarity letter-based decoding criterion was introduced. In addition to the first (exact), second and third ranked letter choices (corresponding to constant chance levels of $3.7 \%, 11.11 \%, 55.55 \%$ ), an alternative decoding criterion was used where additional letter choices were considered among the four letters having the highest similarity (estimated in terms of the temporal correlation coefficient) compared to the ideal response function of the selected letter. The cross-correlations coefficients of the nine different ideal response functions are shown in figure 4. Thereby, if the letter choice were for example the letter ' $\mathrm{D}$ ' the corresponding similarity criterion would additionally consider the letter choices ' $M$ ', ' $V$ ', ' $E$ ' and 'B', where ' $M$ ' and ' $V$ ' are the letters having a different task but the same response function, and the letter's ' $E$ ' and ' $B$ ' are based on the same task and have the two highest correlation coefficients across the nine response functions. If the response functions have the same correlation coefficients, the letter with the closest distance on the visual letter display is considered, e.g. if the letter choice were 'S', also ' $R$ ' would be selected but not ' $U$ '. This criterion produced a chance level of $55.55 \%$. For the performance evaluation according to this criterion, all the additional letters provided by the similarity criterion were considered equally possible alternative choices.

\section{Results}

\subsection{GLM results}

Using the automated ROI selection approach on the GLM maps with empirical responses derived from the selected ROIs, a decoding accuracy of $77.66 \%$ for the first letter choice (chance level: $3.7 \%$, minimum accuracy $14.29 \%$, maximum accuracy $100 \%$, SD 23.31) was achieved by fully automating the selection based on the localizer session. Taking into account the 


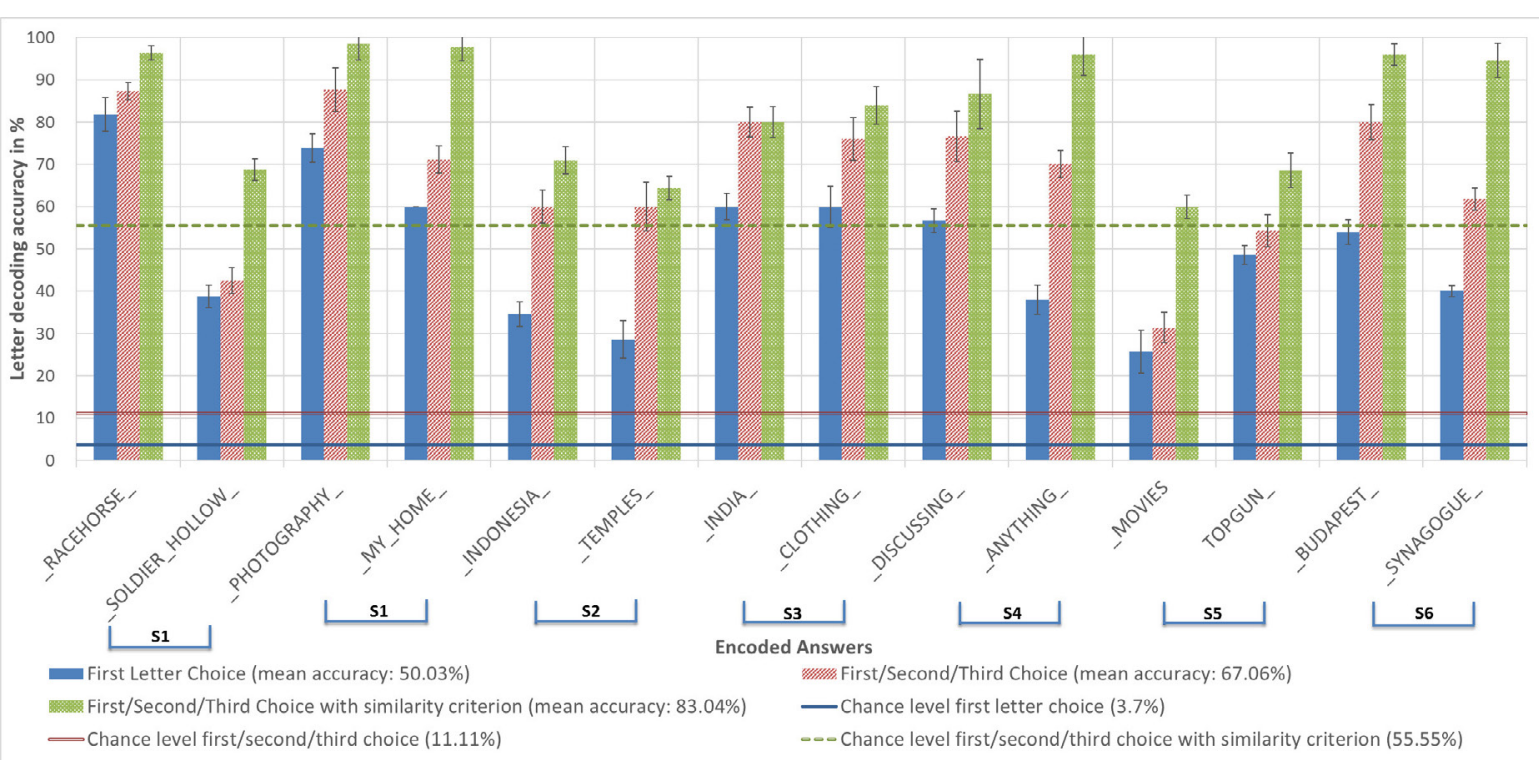

Figure 6. Letter decoding results of ICA-based approach. For each participant, the average decoding accuracy across all letters of the two individually decoded words is shown including SE. The ideal hemodynamic reference functions were used in the shown results.

first, second and third letter choice an accuracy of $92.75 \%$ was reached (chance level: $11.11 \%$, minimum accuracy $57.14 \%$, maximum accuracy $100 \%$, SD 15.36). Considering the similarity selection criterion gave a mean accuracy of $97.31 \%$ (chance level: $55.55 \%$, minimum accuracy $85.71 \%$, maximum accuracy $100 \%$, SD 4.37). The results of each single participant and word are presented in figure 5.

With the ideal response functions, the mean accuracy was $76.81 \%$ for the first letter choice (minimum accuracy $14.29 \%$, maximum accuracy 100\%, SD 24.51), $91.38 \%$ for the first, second and third letter choice (minimum accuracy $70 \%$, maximum accuracy $100 \%$, SD 12.01 ) and $95.82 \%$ for the similarity selection criterion (minimum accuracy $85.71 \%$, maximum accuracy $100 \%$, SD 9.04).

Using the ROIs combined from all participants (excluding the participant under investigation) resulted for the first letter choice in a mean accuracy of $35.94 \%$ (chance level: $3.7 \%$, minimum accuracy $0 \%$, maximum accuracy $77.78 \%$, SD 23.31 ), for the first, second and third letter choice a mean accuracy of $58.66 \%$ was reached (chance level: $11.11 \%$, minimum accuracy $28.57 \%$, maximum accuracy $100 \%$, SD 23.32). The similarity criteria resulted in a mean accuracy of $81.93 \%$ (chance level: $55.55 \%$, minimum accuracy $54.55 \%$, maximum accuracy $100 \%$, SD 14.58).

\subsection{ICA results}

Using the proposed ICA approach, the letter choices of fourteen different words encoded by six healthy participants were decoded without (manually or automatically) defining ROIs in advance.

For the first letter choice, a mean decoding accuracy was reached of $50.03 \%$ (chance level: $3.7 \%$, minimum accuracy $25.71 \%$, maximum accuracy $81.82 \%$, SD 16.65). For the first, second and third letter choices a mean decoding accuracy was reached of $67.06 \%$ (chance level: $11.11 \%$, minimum accuracy $31.43 \%$, maximum accuracy $87.27 \%$, SD 16.48). Considering the similarity selection criterion, a mean decoding accuracy was reached of $83.04 \%$ (chance level: $55.55 \%$, minimum accuracy $60 \%$, maximum accuracy $98.46 \%$, SD 14.04). The results of each single participant and word are presented in figure 6. In all cases, the use of ideal hemodynamic reference functions and localizer GLM maps yielded better performances compared to using individual empirical responses and ICA maps created from the localizer (step three to five) (first letter choice: $45.04 \%$, first, second and third letter choice: $63.69 \%$, first, second, third and similarity criterion $80.28 \%$ ). Figure 8 shows the difference between the GLM-based localizer map and an ICA-based map selected for decoding, as well as their respective overlap, for two cases of correct decoding of a single letter, respectively in a participant with a good overall performance (and higher overlap for this case) and in a participant with a low mean performance (and lower overlap in this case).

Using not the individual participant GLM localizer maps but the template GLM localizer map obtained from the mean of all participants (except the one currently processed) produced an accuracy of $40.25 \%$ (chance level: $3.7 \%$, minimum accuracy $11.43 \%$, maximum accuracy $76.92 \%$, SD 17.71). For the first, second and third letter choice a mean accuracy of $54.06 \%$ was reached (chance level: $11.11 \%$, minimum accuracy $14.29 \%$, maximum accuracy $92.31 \%$, SD 22.76). The similarity criterion resulted in a mean accuracy of $76.53 \%$ (chance level: $55.55 \%$, minimum accuracy $28.57 \%$, maximum accuracy $100 \%$, SD 16.43).

An overview of the mean accuracies of both methods is shown in figure 7.

\section{Discussion}

Here we have presented, and explored the performances of, a novel mechanism for automated GLM-based ROI selection and introduced the use of real-time ICA in fMRI-based motorindependent communication BCIs. 


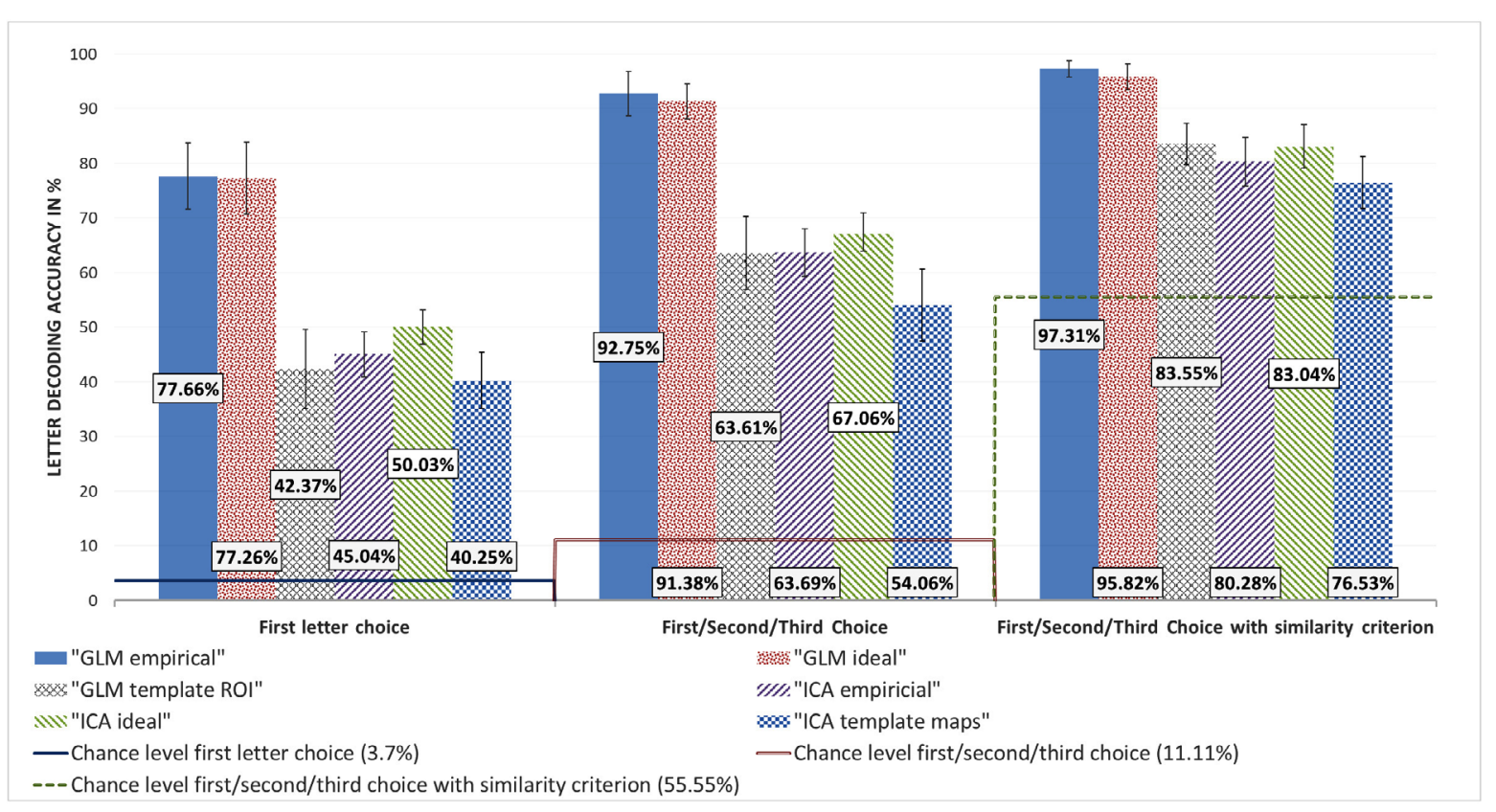

Figure 7. Overview of GLM- and ICA-based encoding results in percentage including SE.

We had two main objectives in mind: (i) proposing an automated, yet stable and flexible, hard thresholding mechanism for univariate GLM modeling of localizer data sets for possible use in GLM-based letter-by-letter decoding, without the need of cognitive network expertise related to the specific chosen mental tasks, and (ii) evaluating the decoding performances of a multi-dimensional data projection method like ICA that can work in real time but is not based on compact ROI pre-selection, and therefore avoids the setting of a voxellevel threshold.

As a target application for the introduced methods, we used the rt-fMRI communication BCI presented in [9]. In this BCI the subject encodes a single letter during each trial and the total time needed to spell a complete word can be up to 10$20 \mathrm{~min}$ (i.e. the number of letters in the word times the total trial duration including the rest period). As this is a common duration for a typical fMRI experimental session, in practice, this BCI can be used to respond to a single question (posed by the researcher) that accepts a single (long) word, or pairs of short words with a preposition or conjunction in between, as a possible answer.

\subsection{Accuracy of the automated GLM-based ROI selection}

The automated GLM-based ROI selection approach showed highly similar accuracies compared to those reached by the previous expert-guided ROI selection approach $(76 \%$ versus $82 \%$ in the first letter choice [9]). Note that this performance was obtained without the use of prior spatial (anatomical or functional) information about the location of the ROIs. In fact, only geometric parameters are set for the ROI searching algorithm that were chosen empirically. More specifically, we found that selecting ROIs with at least 20 voxels in each clusters produced stable results across all participants. This choice was inspired by previous work [9], where a total of six ROIs (two ROIs per task) were manually defined for supervised letter decoding, and each ROI comprised four contiguous voxels within a certain fMRI slice (thereby, the total number of voxels that were a priori selected for decoding was 24). In order to be in line with previous choice (and allow a better comparison between supervised and unsupervised approaches), we decided to set the minimum cluster size to 5 voxels and the minimum total number of activated voxels to 20 voxels. Because the ROIs are not manually defined in the unsupervised approaches (i.e. the ROIs can in principle appear in any location of the brain), we intended to be slightly more conservative for the minimum cluster size ( 5 instead of 4 voxels) and allow for potentially more clusters selected (4 instead of 2 clusters in the specific case of clusters with minimal size). In general, we believe this choice was not overfitted to our data set because the initial choice was not based on specific calculations or statistics performed on the data sets but rather on a priori considerations made (and used) in previous work. On the other hand, when we used the same thresholds for a data set not included in the previous study (namely first session of S1), we obtained similar decoding performances compared to most of the other data sets. Future studies could systematically explore the effect of the number of selected voxels to determine more criterions for optimal static or dynamic voxel selection thresholds. In some individual cases, little adjustments of these parameters lead to better performances (not reported), suggesting that possible improvements in this procedure are still possible. Future work is needed to explore the impact and variability of these parameters on the automatic GLM decoding performances also in other information-encoding paradigms. We also found that, for this approach, using empirically derived hemodynamic reference functions should be preferred albeit the difference, compared to [9], was rather small. This is still in line with earlier studies showing that there is a high variability of the single-trial BOLD responses across different individuals and different brain regions [29, 30]. 
To explore the performances of the GLM-based decoding in a hypothetical localizer-free experimental session, we also replaced the ROIs automatically obtained from the individual localizer data with a different set of ROIs obtained from the localizer data of all other participants, however, the results based on these group-representative template ROIs were considerably worse than those obtained with individual localizers. This might be due to the small number of participants studied that could have determined a low degree of generalization of the calculated group ROIs, or to the fact that using individual ROIs always lead to better results and higher decoding accuracies in general. Further studies involving a considerably higher number of participants have to be performed in order to systematically investigate the potential of this approach. Also, the difference of subject S2 performing mental singing and not inner speech could have lead to a different network activity pattern and therefore to suboptimal results.

More in general, when a BCI or neurofeedback experiment makes use of predefined ROIs, as in the reported templatebased approach, the accuracy also relies on the quality of the registration between the functional data of the specific session and the anatomical or functional data in standardized space for the used ROIs. Thereby, the use of alternative registration algorithms, such as, e.g. cortex-based alignment (CBA) [31] or boundary based registration (BBR) [32] procedures, may result in different accuracies, and must be further investigated to get more insight about which registration procedure is most suitable for BCI and neurofeedback experiments.

As letters that appear close in the display (visual space) do not necessarily correspond to the most similar ideal responses (response space), we also introduced a novel similarity selection criterion to handle alternative choice options based on the measured BOLD responses. This criterion allowed reaching a mean accuracy of $97.31 \%$ across all participants and a maximum accuracy of $100 \%$ in some participants, against a chance of $55.55 \%$. Not only this criterion expands the potential use of the present BCI, e.g. in possible combination with word prediction tools like the so called 'Text on 9 keys' or 'T9' used in mobile phones or similar solutions, but also allowed a more detailed investigation about how the task selection itself is affected by (slightly) wrong results in single steps of the procedure which would be otherwise not visible by only investigating the first choice results.

Although the results were overall encouraging, some variability in the performances were observed and for one of the experimental participants (S5), performances were substantially worse than the others. This variability could be in part explained by the fact that, among all participants, subject S5 was the only one with merely one previous fMRI session, whereas all the others had three or (many) more previous fMRI sessions (see table 1). However, overall, we did not find a significant correlation between the letter performances and the number of previous fMRI sessions, suggesting that already two or three sessions could suffice for a subject to become accustomed with the BCI. Nonetheless, a closer inspection of the results suggested that some of the selected BCI regions turned out to be not strongly enough related to any of the mental tasks during the encoding runs. Although this might be an isolated case, the
ROI selection of areas completely unrelated to the areas initially expected to be involved in the mental task, occurred in another participant as well (albeit with less impact on the final performances), suggesting that the localizer run was probably suboptimal for both these participants and therefore this might be an important aspect, especially for the GLM-based decoding approach that strongly relies on the localizer data quality. In general, a participant needs to be able to perform the mental tasks well and reliably enough to allow sufficient statistical contrast among the different tasks and to ensure that the selection of the different ROIs is sufficiently related to each task, otherwise additional quality measures and spatial guides for ROI selection procedures are needed. For instance, the effect of poor localizer performances on the ROI selection procedure could be potentially mitigated by combining the functional information available from the GLM with atlas-derived templates of the expected location for the brain regions. This could give a better generalization of the calculated beta weights in task-specific areas compared to other brain areas.

It would be in principle possible to replace the proposed GLM-based decoding with multi-voxel pattern analysis (MVPA) models and implement a categorical classification based on local multivariate patterns. However, this would not necessarily improve the communication performances when the errors are due to poor localizer results. In fact, MVPA techniques also rely on the selection of compact ROI(s) from the localizer session and additionally require long training sessions for multi-class classification [33], thereby, their application to a 27-class problem would easily become computationally prohibitive and would therefore make us depart from the main objective of simplifying and automatizing the present communication BCI.

\subsection{Accuracy of the real-time ICA approach}

With multivariate, not local, but whole-brain distributed, and fully data-driven approaches like ICA, the selection of specific brain areas for each task is not necessary, albeit the problem is shifted to the (real-time) selection of task-specific patterns. In this study a sliding window of 40 time points was used to estimate ICA components and to select the component of choice in the signal. It has been previously shown that whenever a task-related component is clearly dominant in the signal, this also results in comparable accuracy between realtime ICA and GLM [16]. However, at the end of our simulated real-time analysis, the average accuracy of the real-time ICA approach across all letters was overall lower compared to the GLM ( $50.03 \%$ compared to $\sim 77.66 \%)$. This can be possibly explained by the fact that the parameters of the experiment were purposely designed to strengthen the GLM decoding approach in the first place. For instance, using more mental tasks producing spatially non-overlapping patterns and less (or no) constrains on the timing control of the task performance, would possibly favor the ICA approach, given that the letter choice strongly relies on the spatial patterns in the first part of the pipeline. Nevertheless, the results clearly suggest that ICA performs significantly above chance level already for the first letter choices (50.03\%, chance level: $3.7 \%$ ), and can 

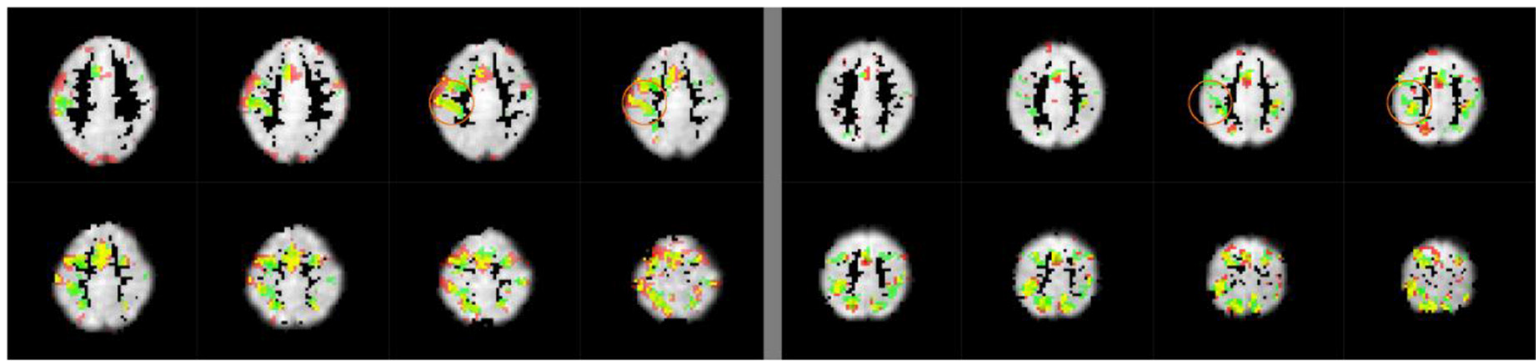

Figure 8. Maps of successful single-trial letter decoding results using ICA. Left panel: participant with high average accuracy (S1). Right panel: participant with low average accuracy (S5). The GLM-derived localizer map is overlaid in green, whereas the selected ICA map for this trial is overlaid in red. The overlap between localizer maps and ICA maps is overlaid in yellow. A circle highlights a brain region in the right prefrontal cortex where the selected ICA map overlaps well (S1) or not well (S5) with the GLM-based localizer map.

reach more than $80 \%$ success in combination with the similarity selection criterion (chance level: $55.55 \%$ ).

For this approach we did not find that, using empirically derived hemodynamic reference functions gives better performances as in the case of the GLM based approach. This can be explained by the fact that ICA component time course is not based on a single area but on a distributed network of areas, and this has the effect of reducing the variability of the single-trial BOLD responses due to the (weighted) averaging process implied by the real-time ICA decomposition $[29,30]$.

Moreover, it turned out that only small benefit resulted from using individual localizer information in the ICA component selection compared to the GLM based method, and that using templates instead of individual localizers made ICA-based decoding slightly outperform GLM-based decoding. This indicates that while using localizer data remains the best option for reaching optimal performances with both the GLM- and ICA- decoding approaches, the ICA-based decoding approach seems to have higher potential for working with predetermined template maps in a possible localizer-free session. This is because the final selection of the best-decoding ICA component at each letter is mainly determined by the intrinsic signal-to-noise of the spatial pattern of activity and to a lesser extent by the precise overlap between the component and the localizer maps (as illustrated in figure 8), and this signal-to-noise depends on the trade-off existing between temporal (i.e. how many time points) and spatial (i.e. how many voxels) data dimensions for the ICA decomposition [16].

In general, the temporal dimension of ICA decompositions is to be necessarily adapted to the single-block duration (in this case it was adapted to the single letter encoding). In fact, while shorter time windows do not necessarily impair the possibility to successfully decompose the data in real-time into meaningful spatial patterns, thereby providing the correct ROIs for task selection via spatial correlation, the component time-course associated with the selected component will be necessarily truncated, and this will likely impair the response selection, which is based on temporal correlations. In line with previous work [16], some preliminary simulations with a localizer data set (not shown) suggested that real-time ICA can often capture signal variations in time windows as short as the rising or falling time of the BOLD response, which is in the order of the time-to-peak of a typical hemodynamic response function (4-8 s, 2-4 TRs). However, when the time window is set to include only a few time points, the latencies of the component time-course cannot be unambiguously decoded due to the absence of sufficient baseline (or plateau) points. It is therefore to be expected, also in different experimental designs, that the minimum duration of the time window for real-time ICA cannot be less than 20-25 s, i.e. the minimum duration of a BOLD 'impulse' response.

Other work on real-time ICA has mainly focused on the performances of different publicly available ICA algorithms applied in sliding window [34] and different procedures to monitor in real-time a target component [35]. In both studies, real-time ICA was applied to simulated real-time fMRI experiments using real fMRI data. By comparing our current implementation to these works, we noted that the fastICA algorithm [27], that we currently use for real-time ICA, was found to be one of the four best performing algorithms among fourteen different algorithms [34]. Moreover, among different procedures for selecting a target component of interest, a 'backprojection' method deriving static spatial information from the functional localizer using off-line ICA, was found to outperform other approaches for dynamic component selection during the simulated real-time acquisition [35]. This approach is very similar to our current implementation, as we also perform off-line ICA on the localizer data sets to generate three task-specific target components, to which the real-time components are spatially correlated.

Potential improvements of the ICA-based method are also possible in relation to the selection of the voxels to be included in the real-time calculations (before pattern generation and selection). In the present application, we performed a highly general selection by including all gray matter voxels, as this is known to improve spatial ICA decompositions in general [26]. Additional voxel selection schemes with a better balance between voxels related versus voxels unrelated to the task pattern may further increase the performances, as it has been shown in an off-line ICA application [36]. In future applications, the use of atlas-derived sets of regions or network configurations might be used with the double purpose to define the global voxel space for ICA calculation on the one hand and allow better component selection on the other hand, resulting in more standardized and portable design for 
the fMRI-BCI, where the preparatory phase is ideally completed outside the scanner.

Moreover, in the present application, only task-related ICA components are considered for the automatic letter decoding. In future applications, other components classifiable on-line as specific noise or motion components according to general task-independent spatio-temporal features (see, e.g. [37]) or via on-line estimation of motion parameters, could be useful for a combined real-time ICA-GLM approach where these 'confound' components are regressed out before applying the described GLM approach.

\section{Conclusions}

We demonstrated that a higher level of automation for ROIbased GLM decoding techniques is possible using proper ROI-searching algorithms, and that real-time ICA can potentially reduce the amount and criticality of prior information needed for decoding spatially distributed patterns, in fMRIbased communication BCIs.

There are three practical implications of this study: (i) whenever the localizer is available, the GLM-approach remains the decoding method of choice; (ii) the introduction of novel mechanisms for unsupervised thresholding of localizer maps may considerably reduce the amount of expertise and time spent on trimming the ROIs; (iii) the use of realtime ICA in localizer-free experimental settings can become an option, but, given the current gap observed in the performances between template-based and localizer-based solutions, this could make sense only in particular cases where localizers are not possible because of extremely limited scanning time or critical for any reason, e.g. in the context of diagnostics in patients with disorders of consciousness or generally fast exhaustion of such patients. Nonetheless, we believe that future research and development along these lines may lead to further increasing the level of automation of fMRI-based BCIs. This aspect may turn out to be particularly relevant for brain-based communication when the ability to process cognitive stimuli of varying complexity is challenged by the complete absence of a behavioral response. More in general, motor impairment, mental fatigue, attention disorders and other cognitive problems typically contribute to the difficulty of communication, thereby more flexible and automated communication BCIs might be helpful in restoring some communication ability.

\section{Acknowledgments}

The research leading to these results has received funding from the European Community's Seventh Framework Programme FP7/2007-2013 under grant agreement number PITN-GA-2011-290011. This work was also supported by the Netherlands Organization for Scientific Research (NWO; RUBICON 446-09-010 awarded to BS) and the European Research Council (ERC; advanced grant \#269853 awarded to $\mathrm{RG})$.

\section{References}

[1] Weiskopf N 2012 Real-time fMRI and its application to neurofeedback Neuroimage 62 682-92

[2] Ruiz S, Buyukturkoglu K, Rana M, Birbaumer N and Sitaram R 2014 Real-time fMRI brain computer interfaces: self-regulation of single brain regions to networks Biol. Psychol. 95 4-20

[3] Stoeckel L E et al 2014 Optimizing real time fMRI neurofeedback for therapeutic discovery and development Neuroimage Clin. 5 245-55

[4] Sulzer J et al 2013 Real-time fMRI neurofeedback: progress and challenges Neuroimage 76 386-99

[5] Naci L, Monti M M, Cruse D, Kübler A, Sorger B, Goebel R, Kotchoubey B and Owen A M 2012 Brain-computer interfaces for communication with nonresponsive patients Ann. Neurol. 72 312-23

[6] Sorger B, Dahmen B, Reithler J, Gosseries O, Maudoux A, Laureys S and Goebel R 2009 Another kind of 'BOLD response': answering multiple-choice questions via online decoded single-trial brain signals Prog. Brain Res. 177 275-92

[7] Eklund A, Andersson M, Ohlsson H, Ynnerman A and Knutsson H 2010 A brain computer interface for communication using real-time fMRI Proc.-Int. Conf. on Pattern Recognition pp 3665-9

[8] Yoo S S, Fairneny T, Chen N K, Choo S E, Panych L P, Park H, Lee S Y and Jolesz F A 2004 Brain-computer interface using fMRI: spatial navigation by thoughts Neuroreport 15 1591-5

[9] Sorger B, Reithler J, Dahmen B and Goebel R 2012 A realtime fMRI-based spelling device immediately enabling robust motor-independent communication Curr. Biol. 22 1333-8

[10] Sokunbi M O, Linden D E J, Habes I, Johnston S and Ihssen N 2014 Real-time fMRI brain-computer interface: development of a 'motivational feedback' subsystem for the regulation of visual cue reactivity Front. Behav. Neurosci. 8392

[11] Koush Y, Meskaldji D-E, Pichon S, Rey G, Rieger S W, Linden D E J, Van De Ville D, Vuilleumier P and Scharnowski F 2015 Learning control over emotion networks through connectivity-based neurofeedback Cereb. Cortex 2015 bhv311

[12] Lorenz R, Monti R P, Violante I R, Anagnostopoulos C, Faisal A A, Montana G and Leech R 2016 The automatic neuroscientist: a framework for optimizing experimental design with closed-loop real-time fMRI Neuroimage 129 320-34

[13] Cohen Kadosh K, Luo Q, de Burca C, Sokunbi M O, Feng J, Linden D E J and Lau J Y F 2016 Using real-time fMRI to influence effective connectivity in the developing emotion regulation network Neuroimage 125 616-26

[14] Naci L, Cusack R, Jia V Z and Owen A M 2013 The brain's silent messenger: using selective attention to decode human thought for brain-based communication J. Neurosci. 33 9385-93

[15] Nelder J A and Wedderburn R W M 1972 Generalized linear models J. R. Stat. Soc. A 135 370-84

[16] Esposito F, Seifritz E, Formisano E, Morrone R, Scarabino T, Tedeschi G, Cirillo S, Goebel R and Di Salle F 2003 Realtime independent component analysis of fMRI time-series Neuroimage 20 2209-24

[17] Megan T, Cohen J D, Lee R F, Norman K A and Turk-browne N B 2015 Closed-loop training of attention with real-time brain imaging Nat. Neurosci. 18 1-9

[18] Oldfield R C 1971 The assessment and analysis of handedness: the Edinburgh inventory Neuropsychologia 9 97-113 
[19] Posse S et al 2000 A new approach to measure single-event related brain activity using real-time fMRI: feasibility of sensory, motor, and higher cognitive tasks Hum. Brain Mapp. 12 25-41

[20] Lorenz R, Pascual J, Blankertz B and Vidaurre C 2014 Towards a holistic assessment of the user experience with hybrid BCIs J. Neural Eng. 1135007

[21] Reuderink B, Nijholt A and Poel M 2009 Affective Pacman: a frustrating game for brain-computer interface experiments Lecture Notes of the Institute for Computer Sciences (Social-Informatics and Telecommunications Engineering) ed A Nijholt et al (Berlin: Springer) pp 221-7

[22] Farwell L A and Donchin E 1988 Talking off the top of your head: toward a mental prosthesis utilizing event-related brain potentials Electroencephalogr. Clin. Neurophysiol. 70 510-23

[23] Sedgewick R 1990 Algorithms in C (Boston, MA: Addison-Wesley Longman Publishing Co., Inc.)

[24] Friston K J, Fletcher P, Josephs O, Holmes A, Rugg M D and Turner R 1998 Event-related fMRI: characterizing differential responses Neuroimage 7 30-40

[25] Goebel R, Esposito F and Formisano E 2006 Analysis of functional image analysis contest (FIAC) data with brain voyager QX: from single-subject to cortically aligned group general linear model analysis and self-organizing group independent component analysis Hum. Brain Mapp. 27 392-401

[26] Formisano E, Esposito F, Di Salle F and Goebel R 2004 Cortex-based independent component analysis of fMRI time series Magn. Reson. Imaging 22 1493-504

[27] Hyvarinen A 1999 Fast and robust fixed-point algorithm for independent component analysis IEEE Trans. Neural Networks 10 626-34

[28] Misaki M, Barzigar N, Zotev V, Phillips R, Cheng S and Bodurka J 2015 Real-time fMRI processing with physiological noise correction-comparison with off-line analysis J. Neurosci. Methods 256 117-21
[29] Handwerker D A, Ollinger J M and D’Esposito M 2004 Variation of BOLD hemodynamic responses across subjects and brain regions and their effects on statistical analyses Neuroimage 21 1639-51

[30] Aguirre G K, Zarahn E and D'esposito M 1998 The variability of human, BOLD hemodynamic responses Neuroimage $8360-9$

[31] Frost M A and Goebel R 2012 Measuring structural-functional correspondence: spatial variability of specialised brain regions after macro-anatomical alignment Neuroimage 59 1369-81

[32] Greve D N and Fischl B 2009 Accurate and robust brain image alignment using boundary-based registration Neuroimage 48 63-72

[33] Stokes M, Thompson R, Cusack R and Duncan J 2009 Top-down activation of shape-specific population codes in visual cortex during mental imagery $J$. Neurosci. 29 1565-72

[34] Soldati N, Calhoun V D, Bruzzone L and Jovicich J 2013 ICA analysis of fMRI with real-time constraints: an evaluation of fast detection performance as function of algorithms, parameters and a priori conditions Front. Hum. Neurosci. 719

[35] Soldati N, Calhoun V D, Bruzzone L and Jovicich J 2013 The use of a priori information in ICA-based techniques for real-time fMRI: an evaluation of static/dynamic and spatial/ temporal characteristics Front. Hum. Neurosci. 764

[36] Aragri A, Scarabino T, Seifritz E, Comani S, Cirillo S, Tedeschi G, Esposito F and Di Salle F 2006 How does spatial extent of fMRI datasets affect independent component analysis decomposition? Hum. Brain Mapp. 27 736-46

[37] De Martino F, Gentile F, Esposito F, Balsi M, Di Salle F, Goebel R and Formisano E 2007 Classification of fMRI independent components using IC-fingerprints and support vector machine classifiers Neuroimage 34 177-94 